\title{
Patterning and polarization of cells by intracellular flows
}

\author{
Rukshala Illukkumbura ${ }^{\mathrm{a}}$, Tom Bland $\mathrm{d}^{\mathrm{a}, \mathrm{b}}$, Nathan W. Goehringa,b,c
}

${ }^{a}$ The Francis Crick Institute, London, UK

${ }^{b}$ Institute for the Physics of Living Systems, University College London, London, UK

${ }^{c}$ MRC Laboratory for Molecular Cell Biology, University College London, London, UK

\begin{abstract}
Beginning with Turing's seminal work [1], decades of research have demonstrated the fundamental ability of biochemical networks to generate and sustain the formation of patterns. However, it is increasingly appreciated that biochemical networks both shape and are shaped by physical and mechanical processes $[2,3,4]$. One such process is fluid flow. In many respects, the cytoplasm, membrane and actin cortex all function as fluids, and as they flow, they drive bulk transport of molecules throughout the cell. By coupling biochemical activity to long range molecular transport, flows can shape the distributions of molecules in space. Here we review the various types of flows that exist in cells, with the aim of highlighting recent advances in our understanding of how flows are generated and how they contribute to intracellular patterning processes, such as the establishment of cell polarity.
\end{abstract} (Word Count: 3200)

Keywords: advection, cortical flow, membrane flow, actomyosin, cell polarity, selforganization 


\section{Introduction}

Cells utilize numerous mechanisms of intracellular transport to drive their spatial organization. Common classes include motor-driven transport along cytoskeletal tracks [5] and diffusion-driven processes in which concentration gradients drive net material flux [6], both of which are implicated in the process of intracellular patterning. Spatially regulated changes in diffusion, for example due to localized membrane binding or phase separation, can drive net diffusive flux and local accumulation [7, 8]. Similarly, spatial bias in the polarity of cytoskeletal tracks or of the rates of motor binding and unbinding can drive directed cargo transport [7, 9].

Here we focus on a class of transport mechanisms defined by advection - the transport of molecules or organelles by bulk flows - and consider their role in intracellular patterning and cell polarity. Analogous to the trans port of cargo by ships traveling through the ocean versus objects carried by ocean currents, motor-based transport along cytoskeletal tracks moves cargo through a medium, e.g. the cytoplasm, while in advection molecules are transported through the cell by the motion of the medium itself. In this review, we will consider the various types of flows observed in cells and the mechanisms by which they are generated, followed by an exploration of how molecules tap into these flows and consequently how cells can utilize advection to drive spatial organization, with an emphasis on symmetry-breaking and cell polarity.

\section{How do cells generate intracellular flows?}

Treadmilling is a central feature of both microtubules and actin filaments. Both grow by subunit addition, and due to their polar nature, this growth occurs preferentially at one end, balanced by disassembly at the other [9]. If a filament is elongating against a barrier, addition at the growing end results in a rearward flux of subunits [10]. This simple conveyor-belt like motion allows transport of molecules associated with the rearward-moving subunits and has been implicated in transport of signaling receptors within filopodia [11], intracellular virus particle trafficking $[12,13]$ and organelle partitioning [14] (Figure 1A).

In most cells, however, cytoskeletal flows do not rely on the treadmilling of isolated 
filaments, but on long range flows of cytoskeletal networks. The C. elegans zygote, for example, exhibits flows of a highly-crosslinked, membrane-associated, contractile actomyosin network [15] (Figure 1B). Cortical flows promote symmetry-breaking along the anterior-posterior axis through the advection of polarity components [16] and are powered by a contractile asymmetry resulting from the polarized distribution and activity of non-muscle myosin (NMY-2) [17, 18]. Local network contraction both pulls actin networks along the membrane towards the anterior while simultaneously accelerating local disassembly and turnover via increased local network stress, resulting in long range flow of material toward the anterior $[17,19,20]$. Similar cortical actin flows are prominent during cell division, where they are directed towards the ingressing cytokinetic furrow, promoting local alignment of actin filaments and flux of actomyosin material into the cleavage furrow to aid cytokinetic ring constriction $[21,22,23]$ as well as within the lamellipodia of migrating cells, where it is referred to as 'retrograde flow' [24]. In certain cases of amoeboid cell migration, the entire cortex appears to flow rearwards [25, 26, 27].

Membranes are also thought to be capable of undergoing flow, with tension gradients inducing flows of membrane lipids. Lipid flow can be induced by artificially applied tension, e.g. via micropipette [28], and is fueled by processes such as membrane protrusion or spatially separated zones of exo- and endocytosis in cells [29, 30, 31]. Long-range lipid flows have been proposed to under lie cell migration via a conveyor-belt like process [29] (Figure 1C). Although there is evidence for flows of membrane components in some contexts [32] and disruption of trafficking impedes motility in several systems [33, 34], the notion of such a 'fluid drive' is controversial $[35,36]$. Lipid flow has also been proposed to account for long-range communication of mechanical cues across the length of the cell through propagation of membrane tension $[30,31]$. However, recent measurements revealed that tension fails to propagate over distances greater than $5 \mu \mathrm{m}$ [37], suggesting that the situation in cells is more complex, most likely due to the large numbers of transmembrane proteins that are immobilized through connections to the underlying actin cortex or external matrix which would hinder lipid flow [38, 39].

Finally, any discussion of intracellular flows would be remiss not to include cytoplasmic flows, also known as cytoplasmic streaming or cyclosis. Cytoplasmic flows often originate 
from cytoskeletal activity at the cell cortex, including motor-dependent transport of vesicles/organelles along cytoskeletal tracks [40] or from cortical actin flow [41] that entrain the motion of the surrounding cytoplasm. Flows can also arise from pressure gradients driven by applied stress, for example actomyosin contraction, cell shape deformation, or osmotic gradients [42, 41]. Because the cell is generally considered a closed volume, cytoplasmic flows typically lead to mixing much like stirring a pot. Advective mixing is likely to be critical in large cells, where random diffusion is insufficient to allow nutrients, proteins, and other factors to mix throughout the cell interior [43]. For example a $30 \mathrm{kDa}$ monomeric protein that diffuses across the length of $E$. coli in $10 \mathrm{~ms}$, would take approximately 5 hours to traverse the $1 \mathrm{~mm}$ Xenopus egg [44]. Although cytoplasmic flows are directly implicated in cell polarity and symmetry-breaking in several contexts (see $A$ role for flows in polarity), on their own they tend to reduce rather than enhance molecular asymmetries in the cell.

\section{What exactly is flowing?}

Having established that cells generate flows, how do cells use flows to drive intracellular transport and the local concentration of molecules in space? To understand this, we must first consider what exactly is flowing.

For a flowing actin cortex, it is clear that the movement of components physically connected to actin filaments or directly incorporated into the actin network will be moved with the same velocity as the cortex. But what about molecules not directly bound to the actin network? Some structures, such as microtubules, intermediate filaments, and organelles, are entangled by the actin meshwork and efficiently transported by retrograde actin flows [45]. It also seems likely that the $10-20 \%$ of membrane embedded proteins that are physically linked to the actin cortex will move through the membrane like a rake, potentially entraining the motion of the surrounding membrane components, while also limiting the ability of lipids to flow independently from the underlying cortex [46, 28, 41]. Thus, in an intact cell, the membrane and actin cortex are perhaps best treated as a form of composite, hydrodynamically-coupled material rather than as isolated, independently behaving structures [37, 47] (Figure 2A). 
The cortex is also permeated by cytosol. Beyond length scales of a micron and for timescales typical of cortical flow, the cytoplasm can be considered viscous. Thus, shear stresses produced by motion of a membrane-associated cytoskeletal-motor network will tend to entrain the motion of the surrounding cytoplasm and objects embedded within it, transducing force produced at the cortex deep into the cell interior. Such hydrodynamic models capture the patterns of flows observed in meiotic and mitotic one-cell stage $C$. elegans embryos [48, 49, 50], Drosophila oocytes [51, 52] and plant cells [53], explaining how flow drives long-range transport of cytoplasmic structures. Theory suggests that the membrane bilayer can sustain applied shear force [54], so in principle, cytoplasmic flow could also induce flow in the overlying membrane. Consistent with this theoretical framework, the application of shear flows to the surface of artificial membranes induces lipid flow in the plane of the bilayer $[55,56]$, though the situation in intact cells is likely more complicated [41]. The potential for hydrodynamic coupling is perhaps best exemplified by Characean algae. Here the cytoplasm, the vacuolar membrane and the vacuole interior appear to be hydrodynamically coupled and are all set in motion by myosin motors moving along cortical actin bundles, yielding rapid long-range internal fluid flows critical for the transport of metabolites in these extremely large cells [57, 58, 59] (Figure 2B).

At the same time, in the complexity of the cellular environment, it is clear that not everything goes with the flow. Beads non-specifically attached to the surface of migrating cells can exhibit mixed behavior with some moving with retrograde flow and others showing unbiased diffusive motion, thus arguing for differential sensitivity to flows [60]. Moreover, attempts to assess this question with fluorescent labeled lipids in a number of systems have failed to reveal flow of membrane lipids despite rapid flow of the underlying cortex $[35,36]$. In the next section we explore some of this complexity by examining the features of molecules that allow them to tap into flows to achieve polarized distributions.

\section{Tapping into flows to generate molecular asymmetries}

Net transport of molecules in the cell depends not only on the pattern of flows, but also on the relative contributions of directional transportversus diffusive or random motion. This ratio 
of advective vs. diffusion-like motion can be captured by the dimensionless Péclet number defined as $P e=L u / D$, where $L$ is the typical length scale, $u$ is the velocity, and $D$ is the effective diffusion coefficient. Over long distances (large $L$ ) or for slowly diffusing species such as organelles or large macromolecular assemblies (small $D)$, flows dominate $(P e \gg 1)$. By contrast over sufficiently short distances or for rapidly diffusing small molecules, the effects of flow may be negligible $(P e \ll 1)$ (Figure 3A). Given a range of values typical for intracellular motor-dependent transport and protein diffusion $(L=1-10 \mu \mathrm{m}, u=0.1-1 \mu \mathrm{m} / \mathrm{s}$, $D=0.01-10 \mu \mathrm{m}^{2} / \mathrm{s}$ ), one obtains estimates of $P e$ spanning $10^{-2}$ to $10^{3}$. Thus, cells occupy a potential sweet spot for interplay between advective and diffusivetransportallowing them to tune the ability of molecules to be transported by flows by shifting the relevant parameters, but also necessitating a case-by-case evaluation of the Péclet number.

One common way to shift the mobility characteristics of a molecule is throughclustering. First noted in the context of immunoglobulin crosslinking in lymphocytes, clustering of cell surface molecules can give rise to a process termed cell capping in which cross-linked islands of surface molecules flow towards and accumulate at the cell rear [61, 62, 63]. However, the precise mechanisms underlying rearward flow can differ and remain unclear in some systems due to the multifaceted nature of clustering. While reduced diffusion and increased avidity for the membrane or cytoskeletal structures would be expected to favor advection (e.g. higher $P e$ ), other effects are less predictable, including cluster-dependent changes in internalization, conformation or recruitment of modifier proteins.

Ligand-dependent clustering of cell surface receptors, for example of both integrins and EGR receptors, is thought to promote receptor activation and their ability to bind to actin and be transported by actin flows $[64,11]$. Clustering also tends to reduce the diffusion of molecules in the membrane, typically more than might be expected from theoretical predictions based on size. For example, measurement of E-cadherin diffusion reveals 10- to 40-fold decrease in diffusion upon oligomerization [65]. In vivo and in vitro data also support a role for membrane-associated actin in local membrane compart mentalization $[66,67,68$, $69,70]$, which would both reduce diffusion, but also promote flow-dependent transport of molecules trapped within cytoskeletal compartments [71].

The affinity with which molecules associate with moving material is also critical. This 
principle is nicely illustrated by the observation that different actin binding probes acquire varying distributions in the context of an identical polarized actin flow, with higher affinity probes acquiring steeper, more polarized concentration profiles [72, 73]. This difference arises because although all probes switch between a bound state and a freely diffusive state, the difference in affinity changes the relative amount of time spent in the two states. Hence higher affinity probes will be advected further during each binding event (increased $L$ ) while reducing the time available to diffuse (i.e. reduced effective $D$ ), both of which should increase $P e$. The effect of this shift in the relative contributions of advection and diffusion for differing binding times is shown in Figure 3B. In this context, avidity effects of clustering come into play. Whereas an isolated molecule may only briefly associate with a flowing material, the combination of multiple such molecules into a single large assembly can dramatically increase the effective lifetime of the bound state.

The dynamic association of clusters of membrane-associated proteins with variable affinities for the actin cortex can give rise to an effective friction, al lowing force transduction from the cortex to membrane-associated molecules and the extracellular environment. At both the immunological synapse and within focal adhesions, affinity differentials of protein complexes yield distinct coupling strengths between molecules and the underlying cortex $[74,75,76]$. At the immunological synapse of $\mathrm{T}$ cells, receptor-crosslinking induces clustering and concentration of receptors at the center of synapse through centripetal flows $[75,76]$. Normally, large clusters are concentrated tightly at the center, with less clustered receptors surrounding the central core. Artificially enhancing clustering of less clustered receptors allowed them to reach the center, consistent with avidity to the cortex enhancing 'frictional coupling' and thereby transport by actin flows. In several systems, molecules undergo phase separation into macroscopic biomolecular condensates, which similarly enables collective transport of otherwise low affinity cortex-binding proteins [77, 78]. Thus, by tuning the biophysical properties of molecules, cells have a rich array of tools to control advective transport.

\section{A role for flows in cell polarity}


Intracellular flows have been documented for well over two centuries [79], and are associated with cell movement [80] and an array of long-range trans port processes [43]. Over the past decade or so, we have come to appreciate the additional role of intracellular flows in orchestrating spatial patterns within the interior of cells. Although the precise role of flows is not always clear, there are now numerous examples of cellular asymmetry associated with intracellular flows.

\subsection{Flows drive active mixing to enable spatial patterning processes}

Some aspects of cortical flows hinge not on their ability to drive net transport on their own, but to enable molecules or intracellular structures to explore the cell. Diffusion and capture processes are common mechanisms for spatially localizing effectors by upstream processes. However, while this works well for small, mobile proteins in relatively small cells $(<10 \mu \mathrm{m})$, diffusion becomes limiting in large cells (large $L)$ or for larger structures, such as vesicles or organelles (low $D$ ). To get around these limitations, cells use active transport to drive mixing throughout the cell interior $[81,82,83,50]$.

In Drosophila melanogaster, a key step in patterning is the localization of specific mRNAs to the anterior and posterior of the oocyte. Evidence supports microtubuledependent trapping of bicoid mRNA at the anterior [84], and actin/myosin V-dependent trapping of oskar mRNA and nanos mRNA at the posterior $[85,86]$. While the contribution of biased microtubule transport (oskar) and simple diffusion (nanos) can support some level of polarity, unbiased but active transport is required to achieve wild-type levels of polarized localization. For posterior mRNAs, microtubule-dependent cytoplasmic streaming facilitates encounters of mRNA with the posterior actin trap [87, 86] (Figure 4A).

This mixing effect can also enable or speed up more complex patterning processes, such as pattern formation by reaction-diffusion, which may also be limited by diffusion in large cells. For example, predicted timescales for the establishment of a stable Bicoid gradient in the Drosophila embryo by diffusion alone have been argued to be too slow, but this timescale is accelerated if the effects of cytoplasmic streaming are included [88]. Thus, active mixing may be a general mechanism for facilitating patterning processes in the cell. 


\subsection{Compartmentalization of cells by flow geometry}

Asymmetries can also emerge from the interplay of flow geometry and cell shape. One example comes from filamentous fungi. Here, cytoplasmic streaming is mediated by an osmotic pressure gradient which generally drives expansion of the fungal hyphal tip [42]. In Neurospora, hyphae are compartmentalized by septa with a central pore, leading to acceleration of the flowing cytoplasm as it passes through. Unexpectedly, microfluidic eddies form in the corners just upstream of the pore. These eddies effectively create a subcellular compartment in which nuclei become trapped, leading to asymmetric nuclear differentiation in individual compartments [89] (Figure 4B).

In animal cells, the actin cortex can generate fountain-head flows of cytoplasm, which are also implicated in asymmetric nuclear positioning. In mammalian oocytes, flows contribute to asymmetric positioning of the mammalian meiotic spindle to facilitate meiotic division [90,91], while in C. elegans, flows drive close apposition of the male pronucleus and its associated centrosomes with the cortex at one end of the ellipsoid zygote to promote polarization $[92,93,94]$.

\subsection{Regulated clustering enables symmetry-breaking by flows}

The C. elegans zygote exemplifies flow-induced symmetry breaking. As noted earlier, cortical flows are stimulated along the nascent anterior-posterior axis, driven by a centrosomeinduced local reduction in actomyosin contractility $[95,17,96]$. These flows polarize the embryo through their ability to drive anterior-directed transport of components of a polarity network known as PAR proteins [15]. Biophysical measurements combined with theory suggest that the diffusion and membrane exchange kinetics of PAR proteins are consistent with advective transport by cortical flow $[16,18]$. More recently, transport of the aPAR proteins was found to rely on cell-cycle dependent clustering of the aPAR protein PAR-3, suggesting its molecular behavior is tuned to facilitate trans port [97, 98, 99]. Single particle tracking is consistent with a clustering-dependent increase in the effective Péclet number through decreasing diffusion and increasing membrane-residency times [98] (Figure 4C).

Similar cortical flows have been implicated in defining polarity domains in Drosophila 
neuroblasts [100, 101] and epithelial cells [102]. Combined with the ability of polarity determinants to form clusters or segregate within phase-separated condensates in diverse systems [103, 104, 105], regulated assembly of higher order structures is emerging as a generic mechanism for asymmetric segregation of specific molecules by polarizing flows.

\subsection{Feedback between actin flow and molecular asymmetry at the leading edge}

In migrating cells, the role of flows is typically discussed in the context of force generation required for cell motility. In this context, polarity is usually considered to be a cause rather than consequence of cell migration [106]. Thus, there has been extensive focus placed on the ability of biochemical networks to drive intracellular patterning. However, mechanical and physical processes are increasingly seen as key contributors, if not equal players in the self-assembly of polarized actin networks in migrating cells [2, 107]. The ability of flows to transport molecules inside the cell means that the very process of generating flows involved in cell motility will necessarily alter the distribution the molecules that guide and enable motility in the first place (Figure 4D). One such molecule is myosin itself, best explored in the context of migration of fish keratinocytes. Keratinocytes are remarkable for their ability to undergo self-organization of their cytoskeleton. Even cell fragments can be induced to polarize and migrate through the application of simple mechanical perturbations [108]. In these cells (and many others), myosin asymmetry simultaneously underlies and is enhanced by long-range actin cortical flows [109]. The resulting flow-contractility positive feedback circuit has been shown to be sufficient for polarization and migration of cells in both theoretical and experimental models $[107,26]$. Because flows would be expected to transport any number of molecules that may regulate polarity and actin flow, the flow-polarity feedback paradigm is likely to be common, a view supported by the observation of a universal relationship between migration speed and cell persistence [72].

It is important, however, to note that not all molecules will be polarized by this mechanism. Some molecules may not undergo rearward transport due to their diffusivity, rapid exchange kinetics, or an inability to couple to flows. However, for molecules that are transported by retrograde flows, the steady-state density of molecules across the cell will 
depend critically not only on the balance of advection and diffusion within the membrane, but also trafficking to and from the plasma membrane (Figure 4D). Rapid recycling will tend to favor enrichment at the leading in the face of flows, as in the case of 120 , which following rearward flow is actively endocytosed and trafficked back to the leading edge to facilitate junction formation during collective cell migration [110]. At the same time, accumulation at the cell rear will tend to require slower turnover rates allows for flow to build up the required concentration gradients. Intriguingly, such slow turnover kinetics have been argued to constitute a directional memory: because any accumulated asymmetry of molecules with low turnover will decay slowly with time, cells will retain a memory of prior inputs in the face of time-varying flow, therefore buffering against rapid variation in input signal [111].

\section{Outlook}

Recent technological advances have enabled dramatic improvement in our ability to visualize, measure and control flows in cells. FRAP (Fluorescence Recovery After Photobleaching), single molecule tracking, FCS (Fluorescence correlation spectroscopy), and image-based flow analysis and visualization tools are revealing increasing detail of the mobility of molecules in their native context [112]. Optogenetics and photo-switchable reagents provide a method for manipulating the pathways that generate flows $[72,33,113]$, while laser-induced thermoviscous fluid flows open the door to manipulating flows independently of a cell's regulatory or cytoskeletal networks [114]. Finally optical, magnetic, and hydrodynamic traps provide an avenue for measuring forces, trapping molecules and/or objects and manipulating their motion [115]. At the same time, synthetic biology approaches using artificial mem branes provide defined minimal systems to explore how the molecular characteristics and membrane architecture affect advective and diffusive transport, while advanced microfabrication technologies allow fine scale manipulation of the membrane surface $[116,117,118]$. We are still ways off from unifying observations drawn from theory and synthetic systems with the complexity of intact cells, but armed with a new generation of tools, the gaps in understanding how cellular flows drive spatial patterning of cells are closing fast. 


\section{Disclosure}

The authors declare no conflict of interest.

\section{Acknowledgements}

The authors would like to thank Guillaume Charras, Rob Arkowitz, and members of the Goehring lab for helpful comments, and Jaipal Dhiman for an engineer's perspective on fluid dynamics. Work in the authors' lab is supported by the Francis Crick Institute, which receives its core funding from Cancer Research UK (FC001086), the UK Medical Research Council (FC001086), and Wellcome Trust (FC001086), and the EU Horizon 2020 research and innovation program under the Marie Skodowska-Curie grant agreement 675407 (PolarNet).

\section{References}

1. Turing, A.M.. The chemical basis of morphogenesis. Philosophical Transactions of the Royal Society of London 1952; 237(641):37-72. doi:10.1007/BF02459572.

2. Goehring, N.W., Grill, S.W.. Cell polarity: Mechanochemical patterning. Trends in Cell Biology 2013; 23(2):72-80. doi:10.1016/j.tcb. 2012.10.009.

3. Howard, J., Grill, S.W., Bois, J.S.. Turing's next steps: the mechanochemical basis of morphogenesis. Nature Reviews Molecular Cell Biology 2011; 12(6):392-398. doi:10.1038/nrm3120.

4. Heisenberg, C.P., Bellaïche, Y.. Forces in tissue morphogenesis and patterning. Cell 2013; 153(5):948. doi:10.1016/j.cell.2013.05.008.

5. Vale, R.D.. The Molecular Motor Toolbox for Intracellular Transport. Cell 2003; 112(4):467-480. doi:10.1016/S0092-8674(03)00111-9.

6. Berg, H.C., Purcell, E.M.. Physics of chemoreception. Biophysical Journal 1977; 20(2):193-219. doi:10.1016/S0006-3495(77)85544-6.

7. Peglion, F., Goehring, N.W.. Switching states: dynamic remodelling of polarity complexes as a toolkit for cell polarization. Current Opinion in Cell Biology 2019; 60:121-130. doi:10.1016/j.ceb.2019.05.002.

8. Wu, Y., Han, B., Li, Y., Munro, E., Odde, D.J., Griffin, E.E.. Rapid diffusion-state switching underlies stable cytoplasmic gradients in the Caenorhabditis elegans zygote. Proceedings of the National Academy of Sciences 2018; 115(36):E8440-E8449. doi:10.1073/pnas.1722162115.

* Using single particle tracking, this study reveals spatially regulated switching in the diffusivity of cytoplasmic fate determinants that is essential for creating polarized gradients in the C. elegans zygote. 
9. Li, R., Gundersen, G.G.. Beyond polymer polarity: How the cytoskeleton builds a polarized cell. Nature Reviews Molecular Cell Biology 2008; 9(11):860-873. doi:10.1038/nrm2522.

10. Hill, T.L., Kirschner, M.W.. Subunit treadmilling of microtubules or actin in the presence of cellular barriers: possible conversion of chemical free energy into mechanical work. Proceedings of the National Academy of Sciences 1982; 79(2):490-494. doi:10.1073/pnas.79.2.490.

11. Lidke, D.S., Lidke, K.A., Rieger, B., Jovin, T.M., Arndt-Jovin, D.J.. Reaching out for signals: Filopodia sense EGF and respond by directed retrograde transport of activated receptors. Journal of Cell Biology 2005; 170(4):619-626. doi:10.1083/jcb.200503140.

12. Chaikeeratisak, V., Khanna, K., Nguyen, K.T., Sugie, J., Egan, M.E., Erb, M.L., Vavilina, A., Nonejuie, P., Nieweglowska, E., Pogliano, K., Agard, D.A., Villa, E., Pogliano, J.. Viral Capsid Trafficking along Treadmilling Tubulin Filaments in Bacteria. Cell 2019; 177(7):1771-1780. doi:10.1016/j.cell.2019.05.032.

** This study uses a combination of live imaging, photobleaching experiments and cryo-electron tomography to demonstrate how Pseudomonas phages facilitate capsid transport from the plasma membrane to the nucleus via tubulin-like filament treadmilling mechanisms.

13. Gardet, A., Breton, M., Trugnan, G., Chwetzoff, S.. Role for Actin in the Polarized Release of Rotavirus. Journal of Virology 2007; 81(9):4892-4894. doi:10.1128/jvi.02698-06.

14. Toro-Nahuelpan, M., Müller, F.D., Klumpp, S., Plitzko, J.M., Bramkamp, M., Schüler, D.. Segregation of prokaryotic magnetosomes organelles is driven by treadmilling of a dynamic actin-like MamK filament. $B M C$ Biology 2016; 14(1):88. doi:10.1186/s12915-016-0290-1.

15. Munro, E., Nance, J., Priess, J.R.. Cortical flows powered by asymmetrical contraction transport PAR proteins to establish and maintain anterior-posterior polarity in the early C. elegans embryo. DevelopmentalCell 2004; 7(3):413-424. doi:10.1016/j.devcel.2004.08.001.

16. Goehring, N.W., Hoege, C., Grill, S.W., Hyman, A.A.. PAR proteins diffuse freely across the anterior-posterior boundary in polarized C. elegans embryos. Journal of Cell Biology 2011; 193(3):583-594. doi:10. 1083/jcb.201011094.

17. Mayer, M., Depken, M., Bois, J.S., Jülicher, F., Grill, S.W.. Anisotropies in cortical tension reveal the physical basis of polarizing cortical flows. Nature 2010; 467(7315):617-21. doi:10.1038/ nature09376.

18. Gross, P., Kumar, K.V., Goehring, N.W., Bois, J.S., Hoege, C., Jülicher, F., Grill, S.W.. Guiding self-organized pattern formation in cell polarity establishment. Nature Physics 2019; 15(3):293-300. doi:10.1038/s41567018-0358-7.

* Through a combination of modeling and experimentalmeasurements, this paper proposes an integrated model of coupled feedback between the PAR polarity network and actomyosin flow, highlighting the subcritical nature of the feedback network.

19. Wilson, C.A., Tsuchida, M.A., Allen, G.M., Barnhart, E.L., Applegate, K.T., Yam, P.T., Ji, L., Keren, K., Danuser, G., Theriot, J.A.. Myosin II contributes to cell-scale actin network treadmilling through network disassembly. Nature 2010; 465(7296):373-377. doi:10.1038/nature08994.

20. McFadden, W.M., McCall, P.M., Gardel, M.L., Munro, E.M.. Filament turnover tunes both force generation and dissipation to control long-range flows in a model actomyosin cortex. PLoS Computational Biology 2017; 
13(12):e1005811. doi:10.1371/journal.pcbi.1005811.

* This study provides an elegant computational framework for understanding how cells control cortical actomyosin flow.

21. Bray, D., White, J.G.. Cortical flow in animal cells. Science 1988; 239(4842):883-888. doi:10.1126/science. 3277283 .

22. Reymann, A.C., Staniscia, F., Erzberger, A., Salbreux, G., Grill, S.W.. Cortical flow aligns actin filaments to form a furrow. eLife 2016; 5:e17807. doi:10.7554/elife.17807.

23. Khaliullin, R.N., Green, R.A., Shi, L.Z., Gomez-Cavazos, J.S., Berns, M.W., Desai, A., Oegema, K.. A positive-feedback-based mechanism for constriction rate acceleration during cytokinesis in Caenorhabditis elegans. eLife 2018; 7. doi:10.7554/elife.36073.

* This study provides evidence that flow-dependent transport of cytokinetic ring components into the cleavage furrow gives rise to a positive feedback loop between furrow ingression speed and furrow-directed cortical flows.

24. Cramer, L.P.. Molecular mechanism of actin-dependent retrograde flow in lamellipodia of motile cells. Frontiers in Bioscience 1997; 2(4):260- 270. doi:10.2741/a189.

25. Bergert, M., Erzberger, A., Desai, R.A., Aspalter, I.M., Oates, A.C., Charras, G., Salbreux, G., Paluch, E.K.. Force transmission during adhesion-independent migration. Nature Cell Biology 2015; 17(4):524- 529. doi:10.1038/ncb3134.

26. Ruprecht, V., Wieser, S., Callan-Jones, A., Smutny, M., Morita, H., Sako, K., Barone, V., Ritsch-Marte, M., Sixt, M., Voituriez, R., Heisenberg, C.P.. Cortical contractility triggers a stochastic switch to fast amoeboid cell motility. Cell 2015; 160(4):673-685. doi:10.1016/ j.cell.2015.01.008.

27. Liu, Y.J., Le Berre, M., Lautenschlaeger, F., Maiuri, P., CallanJones, A., Heuzé, M., Takaki, T., Voituriez, R., Piel, M.. Confinement and low adhesion induce fast amoeboid migration of slow mesenchymal cells. Cell 2015;160(4):659-672. doi:10.1016/j.cell. 2015.01.007.

28. Fogelson, B., Mogilner, A.. Computational estimates of membrane flow and tension gradient in motile cells. PLOS ONE 2014; 9(1):e84524. doi:10.1371/journal.pone.0084524.

29. Bretscher, M.S.. Getting Membrane Flow and the Cytoskeleton to Cooperate in Moving Cells. Cell 1996; 87:601-606.

30. Gauthier, N.C., Fardin, M.A., Roca-Cusachs, P., Sheetz, M.P.. Temporary increase in plasma membrane tension coordinates the activation of exocytosis and contraction during cell spreading. Proceedings of the National Academy of Sciences 2011; 108(35):14467-14472. doi:10.1073/pnas.1105845108.

31. Houk, A.R., Jilkine, A., Mejean, C.O., Boltyanskiy, R., Dufresne, E.R., Angenent, S.B., Altschuler, S.J., Wu, L.F., Weiner, O.D.. Membrane tension maintains cell polarity by confining signals to the leading edge during neutrophil migration. Cell 2012; 148(1-2):175-188. doi:10.1016/j.cell.2011.10.050.

32. Dal, J., Sheetz, M.P.. Axon membrane flows from the growth cone to the cell body. Cell 1995; 83(5):693-701. doi:10.1016/0092-8674(95) 90182-5.

33. O’Neill, P.R., Castillo-Badillo, J.A., Meshik, X., Kalyanaraman, V., Melgarejo, K., Gautam, N.. Membrane 
Flow Drives an Adhesion-Independent Amoeboid Cell Migration Mode. Developmental Cell 2018; 46(1):922. doi:10.1016/j.devcel.2018.05.029.

* This study uses optogenetic activation of RhoA and GPCR signaling to trigger different modes of migration in macrophages in suspension to provide support for a model in which rearward cortical and mem brane flows propel the cell forward through exerting viscous forces on the surrounding fluid.

34. Gras, S., Jimenez-Ruiz, E., Klinger, C.M., Schneider, K., Klingl, A., Lemgruber, L., Meissner, M.. An endocytic-secretory cycle participates in Toxoplasma gondii in motility. PLOS Biology 2019; 17(6):e3000060. doi:10.1371/journal.pbio.3000060.

35. Kucik, D.E., Elson, E.L., Sheetz, M.P.. Cell Migration Does Not Produce Membrane Flow. The Journal of cell biology 1990; 111:1617- 1622.

36. Hons, M., Kopf, A., Hauschild, R., Leithner, A., Gaertner, F., Abe, J., Renkawitz, J., Stein, J.V., Sixt, M.. Chemokines and integrins independently tune actin flow and substrate friction during intranodal migration of T cells. Nature Immunology 2018; 19(6):606- 616. doi:10.1038/s41590-018-0109-z.

37. Shi, Z., Graber, Z.T., Baumgart, T., Stone, H.A., Cohen, A.E.. Cell Membranes Resist Flow. Cell 2018; 175(7):1769-1779. doi:10.1016/j.cell.2018.09.054.

** Using a dual optical tweezer setup to pull multiple membrane tethers, this study provides evidence that changes in membrane tension in intact cells do not propagate beyond the local region.

38. Chein, M., Perlson, E., Roichman, Y.. Flow Arrest in the Plasma Membrane. Biophysical Journal 2019; 117(5):810-816. doi:10.1016/j. bpj.2019.07.001.

39. Jacobson, K., Kapustina, M.. Going with the flow (or not). Biophysical Journal 2019; 117(5):791-792. doi:10.1016/j.bpj.2019.07.049.

40. Goldstein, R.E., van de Meent, J.W.. A physical perspective on cytoplasmic streaming. Interface Focus 2015; 5(4):20150030. doi:10. 1098/rsfs.2015.0030.

41. Mogilner, A., Manhart, A.. Intracellular Fluid Mechanics: Coupling Cytoplasmic Flow with Active Cytoskeletal Gel. Annual Review of Fluid Mechanics 2017; 50(1):347-370. doi:10.1146/ annurev-fluid010816-060238.

42. Abadeh, A., Lew, R.R.. Mass flow and velocity profiles in Neurospora hyphae: Partial plug flow dominates intra-hyphal transport. Microbiology 2013; 159:2386-2394. doi:10.1099/mic.0.071191-0.

43. Pickard, W.F.. The role of cytoplasmic streaming in symplastic transport. Plant, Cell and Environment 2003; 26(1):1-15. doi:10.1046/j. 1365-3040.2003.00845.x.

44. Milo, R., Phillips, R.. Cell biology by the numbers. 2016. ISBN 0815345372. doi:10.5860/choice.196525.

45. Leduc, C., Etienne-Manneville, S.. Regulation of microtubule associated motors drives intermediate filament network polarization. Journal of Cell Biology 2017; 216(6):1689-1703. doi:10.1083/jcb. 201607045.

46. Holifield, B.F., Ishihara, A., Jacobson, K.. Comparative behavior of membrane protein-antibody complexes on motile fibroblasts: Implications for a mechanism of capping. Journal of Cell Biology 1990; 111(6 I):24992512. doi:10.1083/jcb.111.6.2499. 
47. Groves, J.T.. Membrane Mechanics in Living Cells. Developmental Cell 2019; 48(1):15-16. doi:10.1016/j.devcel.2018.12.011.

48. Niwayama, R., Shinohara, K., Kimura, A.. Hydrodynamic prop erty of the cytoplasm is sufficient to mediate cytoplasmic streaming in the Caenorhabiditis elegans embryo. Proceedings of the National Academy of Sciences 2011; 108(29):11900-11905. doi:10.1073/pnas. 1101853108.

49. Niwayama, R., Nagao, H., Kitajima, T.S., Hufnagel, L., Shino hara, K., Higuchi, T., Ishikawa, T., Kimura, A.. Bayesian Inference of Forces Causing Cytoplasmic Streaming in Caenorhabditis elegans Embryos and Mouse Oocytes. PloS one 2016; 11(7):e0159917. doi:10.1371/journal.pone.0159917.

50. Kimura, K., Mamane, A., Sasaki, T., Sato, K., Takagi, J., Ni wayama, R., Hufnagel, L., Shimamoto, Y., Joanny, J.F., Uchida, S., Kimura, A.. Endoplasmic-reticulum-mediated microtubule alignment governs cytoplasmic streaming. Nature Cell Biology 2017; 19(4):399-406. doi:10.1038/ncb3490.

* Using a combination of live cell imaging and theoretical modelling, this study provides evidence for a positive feedback mechanism between the microtubule network and the endoplasmic reticulum in generating meiotic cytoplasmic streaming in C. elegans.

51. Ganguly, S., Williams, L.S., Palacios, I.M., Goldstein, R.E.. Cytoplasmic streaming in Drosophila oocytes varies with kinesin activity and correlates with the microtubule cytoskeleton architecture. Proceedings of the National Academy of Sciences 2012; 109(38):15109-15114. doi:10.1073/pnas.1203575109.

52. Deutsch, J.M., Brunner, M.E., Saxton, W.M.. The mechanics of a microscopic mixer: microtubules and cytoplasmic streaming in Drosophila oocytes. arXiv 2011; 1101(2225):7.

53. Houtman, D., Pagonabarraga, I., Lowe, C.P., Esseling-Ozdoba, A., Emons, A.M., Eiser, E.. Hydrodynamic flow caused by active transport along cytoskeletal elements. EPL 2007; 78(1):18001. doi:10.1209/02955075/78/18001.

54. Noguchi, H., Gompper, G.. Fluid vesicles with viscous membranes in shear flow. Physical Review Letters 2004; 93(25). doi:10.1103/ PhysRevLett.93.258102.

55. Jönsson, P., Beech, J.P., Tegenfeldt, J.O., Hök, F.. Shear-driven motion of supported lipid bilayers in microfluidic channels. Journal of the American Chemical Society 2009; 131(14):5294-5297. doi:10.1021/ ja809987b.

56. Honerkamp-Smith, A.R., Woodhouse, F.G., Kantsler, V., Gold stein, R.E.. Membrane viscosity determined from shear-driven flow in giant vesicles. Physical Review Letters 2013; 111(3). doi:10.1103/ PhysRevLett.111.038103.

57. Kamiya, N., Kuroda, K.. Velocity Distribution of the Protoplasmic Streaming in Nitella Cells. Bot Mag Tokyo 1956; 69(822):544-554. doi:10.15281/jplantres1887.69.544.

58. Nothnagel, E.A., Webb, W.W.. Hydrodynamic models of viscous coupling between motile myosin and endoplasm in Characean algae. Journal of Cell Biology 1982; 94(2):444-454. doi:10.1083/jcb.94.2. 444.

59. Goldstein, R.E., Tuval, I., van de Meent, J.W.. Microfluidics of cytoplasmic streaming and its implications for intracellular transport. Proceedings of the National Academy of Sciences 2008; 105(10):3663- 3667. doi:10.1073/pnas.0707223105. 
60. Sheetz, M.P., Turney, S., Qian, H., Elson, E.L.. Nanometre-level analysis demonstrates that lipid flow does not drive membrane glycoprotein movements. Nature 1989; 340(6231):284-288. doi:10.1038/ 340284a0.

61. Taylor, R.B., Duffus, W.P.H., Raff, M.C., De Petris, S.. Redistribution and pinocytosis of lymphocyte surface immunoglobulin molecules induced by anti-immunoglobulin antibody. Nature New Biology 1971; 233(42):225-229.doi:10.1038/newbio233225a0.

62. Bourguignon, L.Y., Singer, S.J.. Transmembrane interactions and the mechanism of capping of surface receptors by their specific ligands. Proceedings of the National Academy of Sciences of the United States of America 1977; 74(11):5031-5.doi:10.1073/pnas.74.11.5031.

63. Dustin, M.L., Groves, J.T.. Receptor Signaling Clusters in the Immune Synapse. Annual Review of Biophysics 2012; 41(1):543-556. doi:10.1146/annurev-biophys-042910-155238.

64. Felsenfeld, D.P., Choquet, D., Sheetz, M.P.. Ligand binding regulates the directed movement of $\beta 1$ integrins on fibroblasts. Nature 1996; 383(6599):438-440. doi:10.1038/383438a0.

65. Iino, R., Koyama, I., Kusumi, A.. Single molecule imaging of green fluorescent proteins in living cells: Ecadherin forms oligomers on the free cell surface. Biophysical Journal 2001; 80(6):2667-2677. doi:10. 1016/S0006-3495(01)76236-4.

66. Nakada, C., Ritchie, K., Oba, Y., Nakamura, M., Hotta, Y., Iino, R., Kasai, R.S., Yamaguchi, K., Fujiwara, T., Kusumi, A.. Accumulation of anchored proteins forms membrane diffusion barriers during neuronal polarization. Nature Cell Biology 2003; 5(7):626-632. doi:10.1038/ncb1009.

67. Suzuki, K., Ritchie, K., Kajikawa, E., Fujiwara, T., Kusumi, A.. Rapid hop diffusion of a G-protein-coupled receptor in the plasma membrane as revealed by single-molecule techniques. Biophysical Journal 2005; 88(5):3659-3680. doi:10.1529/biophysj.104.048538.

68. Gowrishankar, K., Ghosh, S., Saha, S., Rumamol, C., Mayor, S., Rao, M.. Active remodeling of cortical actin regulates spatiotemporal organization of cell surface molecules. Cell 2012; 149(6):1353-1367. doi:10.1016/j.cell.2012.05.008.

69. Heinemann, F., Vogel, S.K., Schwille, P.. Lateral membrane dif fusion modulated by a minimal actin cortex. Biophysical Journal 2013; 104(7):1465-1475. doi:10.1016/j.bpj.2013.02.042.

70. Vogel, S.K., Greiss, F., Khmelinskaia, A., Schwille, P.. Control of lipid domain organization by a biomimetic contractile actomyosin cortex. eLife 2017; 6:1-16. doi:10.7554/eLife.24350.

71. Ewers, H., Smith, A.E., Sbalzarini, I.F., Lilie, H., Koumoutsakos, P., Helenius, A.. Single-paticle tracking of murine polyoma virus like particles on live cells and artificial membranes. Proceedings of the National Academy of Sciences 2005; 102(42):15110-15115. doi:10. 1073/pnas.0504407102.

72. Maiuri, P., Rupprecht, J.F., Wieser, S., Ruprecht, V., Bénichou, O., Carpi, N., Coppey, M., De Beco, S., Gov, N., Heisenberg, C.P., Lage Crespo, C., Lautenschlaeger, F., Le Berre, M., Lennon-Dumenil, A.M., Raab, M., Thiam, H.R., Piel, M., Sixt, M., Voituriez, R.. Actin flows mediate a universal coupling between cell speed and cell persistence. Cell 2015; 161(2):374-386. doi:10.1016/j.cell.2015.01. 056.

73. Bement, W.M., Leda, M., Moe, A.M., Kita, A.M., Larson, M.E., Golding, A.E., Pfeuti, C., Su, K.C., Miller, 
A.L., Goryachev, A.B., von Dassow, G.. Activator-inhibitor coupling between Rho signalling and actin assembly makes the cell cortex an excitable medium. Nature Cell Biology 2015; 17(11):1471-1483. doi: $10.1038 / \mathrm{ncb} 3251$.

74. Hu, K., Ji, L., Applegate, K.T., Danuser, G., Waterman-Storer, C.M.. Differential transmission of actin motion within focal adhesions. Science 2007; 315(5808):111-115. doi:10.1126/science.1135085.

75. DeMond, A.L., Mossman, K.D., Starr, T., Dustin, M.L., Groves, J.T.. T cell receptor microcluster transport through molecular mazes reveals mechanism of translocation. Biophysical Journal 2008; 94(8):3286-3292. doi:10.1529/biophysj.107.119099.

76. Yu,C.h., Wu,H.J., Kaizuka, Y., Vale, R.D., Groves, J.T.. Altered actin centripetal retrograde flow in physically restricted immunological synapses. PLOS ONE 2010; 5(7):e11878. doi:10.1371/journal.pone. 0011878.

77. Ditlev, J.A., Vega, A.R., Köster, D.V., Su, X., Tani, T., Lako duk, A.M., Vale, R.D., Mayor, S., Jaqaman, K., Rosen, M.K.. A composition-dependent molecular clutch between T cell signaling condensates and actin. eLife 2019; 8:1-44. doi:10.7554/eLife.42695.

* This study shows that the transmembrane adapter protein LAT phase separates with actin binding proteins into condensates allowing its efficiently coupling to centripetal actin flows at the T cell synapse.

78. Schwayer, C. Shamipour, S., Pranjic-Ferscha, K., Schauer, A., Balda, M., Tada, M. Matter, K., Heisenberg, C.P.. Mechanosensation of tight junctions depends on ZO-1 phase separation and flow. Cell 2019; 179, 9370952.e18. doi:10.1016/j.cell.2019.10.006.

*Suggests that phase separation of Zona Occludins promotes their ability to be transported by cortical actin flows.

79. Corti, B.. Osservazione Microscopische sulla Tremella e sulla Circu lazione del Fluido in Una Planto Acquaguola. Lucca, Italy: Appresso Giuseppe Rocchi; 1774.

80. Keren, K., Yam, P.T., Kinkhabwala, A., Mogilner, A., Theriot, J.A.. Intracellular fluid flow in rapidly moving cells. Nature Cell Biology 2009; 11(10):1219-1224. doi:10.1038/ncb1965.

81. Brangwynne, C.P., Koenderink, G.H., MacKintosh, F.C., Weitz, D.A.. Intracellular transport by active diffusion. Trends in cell biology 2009; 19(9):423-7. doi:10.1016/j.tcb.2009.04.004.

82. Verchot-Lubicz, J., Goldstein, R.E.. Cytoplasmic streaming enables the distribution of molecules and vesicles in large plant cells. Proto plasma 2010; 240(1):99-107. doi:10.1007/s00709-009-0088-x.

83. Khuc Trong, P., Guck, J., Goldstein, R.E.. Coupling of active motion and advection shapes intracellular cargo transport. Physical Review Letters 2012; 109(2). doi:10.1103/PhysRevLett.109.028104.

84. Trovisco, V., Belaya, K., Nashchekin, D., Irion, U., Sirinakis, G., Butler, R., Lee, J.J., Gavis, E.R., St Johnston, D.. bicoid mRNA localises to the Drosophila oocyte anterior by random Dynein mediated transport and anchoring. eLife 2016; 1-34.doi:10.7554/eLife.17537.

85. Forrest, K.M., Gavis, E.R.. Live imaging of endogenous RNA reveals a diffusion and entrapment mechanism for nanos mRNA localization in Drosophila. Current Biology 2003; 13(14):1159-1168. doi:10.1016/ S0960- 
9822(03)00451-2.

86. Lu, W., Lakonishok, M., Serpinskaya, A.S., Kirchenbüechler, D., Ling, S.C., Gelfand, V.I.. Ooplasmic flow cooperates with trans port and anchorage in Drosophila oocyte posterior determination. The Journal of Cell Biology 2018; 217(10):3497-3511. doi:10.1083/jcb. 201709174.

** This study demonstrates that random transport by cytoplasmic flow and polar capture by myosin $\mathrm{V}$ acts together with directed transport to promote asymmetric accumulation of RNPs.

87. Krauss, J., López de Quinto, S., Nüsslein-Volhard, C., Ephrussi, A.. Myosin-V Regulates oskar mRNA Localization in the Drosophila Oocyte. Current Biology 2009; 19(12):1058-1063. doi:10.1016/j.cub. 2009.04.062.

88. Hecht, I., Rappel, W.J., Levine, H.. Determining the scale of the Bicoid morphogen gradient. Proceedings of the National Academy of Sciences 2009; 106(6):1710-1715. doi:10.1073/pnas.0807655106.

89. Pieuchot, L., Lai, J., Loh, R.A., Leong, F.Y., Chiam, K.H., Sta jich, J., Jedd, G.. Cellular Subcompartments through Cytoplasmic Streaming. Developmental Cell 2015; 34(4):410-420. doi:10.1016/j. devcel.2015.07.017.

90. Yi, K., Unruh, J.R., Deng, M., Slaughter, B.D., Rubinstein, B., Li, R.. Dynamic maintenance of asymmetric meiotic spindle position through Arp2/3-complex-driven cytoplasmic streaming in mouse oocytes. Nature Cell Biology 2011; 13(10):1252-1258. doi:10.1038/ ncb2320.

91. Uraji, J., Scheffler, K., Schuh, M.. Functions of actin in mouse oocytes at a glance. Journal of Cell Science 2018; 131(22):jcs218099. doi:10.1242/jcs.218099.

92. Bienkowska, D., Cowan, C.R.. Centrosomes can initiate a polarity axis from any position within one-cell $C$. elegans embryos. Current Biology 2012; 22(7):583-589. doi:10.1016/j.cub.2012.01.064.

93. McCloskey, R.J., Kemphues, K.J.. Deubiquitylation Machinery Is Required for Embryonic Polarity in Caenorhabditis elegans. PLoS Genetics 2012; 8(11):e1003092. doi:10.1371/journal.pgen.1003092.

94. Saturno, D.M., Castanzo, D.T., Williams, M., Parikh, D.A., Jaeger, E.C., Lyczak, R.. Sustained centrosomecortical contact ensures robust polarization of the one-cell C. elegans embryo. Developmental Biology 2017; 422(2):135-145. doi:10.1016/j.ydbio.2016.12.025.

95. Cowan, C.R., Hyman, A.A.. Centrosomes direct cell polarity in dependently of microtubule assembly in $C$. elegans embryos. Nature 2004;431(7004): 92-96. doi:10.1038/nature02825.

96. Zhao, P., Teng, X., Tantirimudalige, S.N., Nishikawa, M., Woh land, T., Toyama, Y., Motegi, F.. Aurora-A Breaks Symmetry in Contractile Actomyosin Networks Independently of Its Role in Centrosome Maturation. Developmental Cell 2019; 48(5):631-645. doi:10.1016/j.devcel.2019.02.012.

* This work provides evidence that the Aurora A homolog in C. elegans locally modulates flow to regulate cell polarity.

97. Rodriguez, J., Peglion, F., Martin, J., Hubatsch, L., Reich, J., Hirani, N., Gubieda, A.G., Roffey, J., Fernandes, A.R., St Johnston, D., Ahringer, J., Goehring, N.W.. aPKC Cycles between Functionally Distinct PAR Protein Assemblies to Drive Cell Polarity. Developmental Cell 2017; 42(4):400-415. 
doi:10.1016/j.devcel.2017.07.007.

** This study demonstrates that the anterior PAR proteins in the C. elegans zygote consist of two functionally distinct complexes, one which is dependent on PAR-3 clustering and promotes efficient transport by flows, and another that is dependent on CDC-42 and acts to displace posterior PAR proteins from the anterior of the zygote.

98. Dickinson, D.J., Schwager, F., Pintard, L., Gotta, M., Goldstein, B.. A Single-Cell Biochemistry Approach Reveals PAR Complex Dynamics during Cell Polarization. Developmental Cell 2017; 42(4):416-434. doi:10.1016/j.devcel.2017.07.024.

** Through a combination of single cell biochemistry and particle tracking, the study shows that PAR-3 cluster size is cell-cycle dependent and potentially alters diffusion and membrane lifetime to promote advective transport.

99. Wang, S.C., Low, T.Y.F., Nishimura, Y., Gole, L., Yu, W., Motegi, F.. Cortical forces and CDC-42 control clustering of PAR proteins for Caenorhabditis elegans embryonic polarization. Nature Cell Biology 2017; 19(8):988-995. doi:10.1038/ncb3577.

* This study demonstrates that cortical contractility promotes PAR-3 clustering in the C. elegans zygote, which in turn promotes advective transport.

100. Roubinet, C., Tsankova, A., Pham, T.T., Monnard, A., Causs inus, E., Affolter, M., Cabernard, C.. Spatiotemporally separated cortical flows and spindle geometry establish physical asymmetry in fly neural stem cells. Nature Communications 2017; 8(1):1383. doi:10.1038/s41467-017-01391-w.

101. Oon, C.H., Prehoda, K.. Asymmetric recruitment and actin dependent cortical flows drive the neuroblast polarity cycle. eLife 2019; 8:1-15. doi:10.7554/eLife.45815.

102. Zihni, C., Vlassaks, E., Terry, S., Carlton, J., Leung, T.K.C., Olson, M., Pichaud, F., Balda, M.S., Matter, K.. An apical MRCK driven morphogenetic pathway controls epithelial polarity. Nature Cell Biology 2017; 19(9):1049-1060. doi:10.1038/ncb3592.

103. Beutel, O., Maraspini, R., Pombo-Garcia, K., Martin-Lemaitre, C., Honigmann, A.. Phase Separation of Zonula Occludens Proteins Drives Formation of Tight Junctions. bioRxiv 2019; 589580. doi:10. 2139/ssrn.3362257.

104. Harris, T.J.C.. Protein clustering for cell polarity: Par 3 as a paradigm. F1000Research 2017; 6:1620. doi:10.12688/ f1000research.11976.1.

105. Truong Quang, B.A., Mani, M., Markova, O., Lecuit, T., Lenne, P.F.. Principles of E-cadherin supramolecular organization in vivo. Current Biology 2013; 23(22):2197-2207. doi:10.1016/j.cub.2013. 09.015.

106. Etienne-Manneville, S.. Polarity proteins in migration and invasion. Oncogene 2008; 27(55):6970-6980. doi:10.1038/onc.2008.347.

107. Verkhovsky, A.B.. The mechanisms of spatial and temporal patterning of cell-edge dynamics. Current Opinion in Cell Biology 2015; 36:113- 121. doi:10.1016/j.ceb.2015.09.001.

108. Verkhovsky, A.B., Svitkina, T.M., Borisy, G.G.. Self-polarization and directional motility of cytoplasm. Current Biology 1999; 9(1):11-20. doi:10.1016/S0960-9822(99)80042-6.

109. Svitkina, T.M., Verkhovsky, A.B., McQuade, K.M., Borisy, G.G.. Analysis of the actin-myosin II system in 
fish epidermal keratocytes: Mechanism of cell body translocation. Journal of Cell Biology 1997; 139(2):397415. doi:10.1083/jcb.139.2.397.

110. Peglion, F., Llense, F., Etienne-Manneville, S.. Adherens junc tion treadmilling during collective migration. Nature Cell Biology 2014; 16(7):639-651. doi:10.1038/ncb2985.

111. Prentice-Mott, H.V., Meroz, Y., Carlson, A., Levine, M.A., David son, M.W., Irimia, D., Charras, G.T., Mahadevan, L., Shah, J.V.. Directional memory arises from long-lived cytoskeletal asymmetries in polarized chemotactic cells. Proceedings of the National Academy of Sciences 2016; 113(5):1267-1272. doi:10.1073/pnas.1513289113.

112. Jacobson, K., Liu, P., Lagerholm, B.C.. The Lateral Organization and Mobility of Plasma Membrane Components. Cell 2019; 177(4):806- 819. doi:10.1016/j.cell.2019.04.018.

113. Hoorens, M.W., Szymanski, W.. Reversible, Spatial and Tempo ral Control over Protein Activity Using Light. Trends in Biochemical Sciences 2018; 43(8):567-575. doi:10.1016/j.tibs.2018.05.004.

114. Mittasch, M., Gross, P., Nestler, M., Fritsch, A.W., Iserman, C., Kar, M., Munder, M., Voigt, A., Alberti, S., Grill, S.W., Kreysing, M.. Non-invasive perturbations of intracellular flow reveal physical principles of cell organization. Nature Cell Biology 2018; 20(3):344- 351. doi:10.1038/s41556-017-0032-9.

** This study describes focused light-induced cytoplasmic streaming (FLUCS), a technique that uses a laser to induce thermoviscous fluid flows within the cell.

115. Monachino, E., Spenkelink, L.M., van Oijen, A.M.. Watching cellular machinery in action, one molecule at a time. Journal of Cell Biology 2017; 216(1):41-51. doi:10.1083/jcb.201610025.

116. Jönsson, P., Jönsson, B.. Hydrodynamic Forces on Macromolecules Protruding from Lipid Bilayers Due to External Liquid Flows. Langmuir 2015; 31(46):12708-12718. doi:10.1021/acs.langmuir.5b03421.

117. Biswas, K.H., Groves, J.T.. Hybrid Live Cell-Supported Membrane Interfaces for Signaling Studies. Annual Review of Biophysics 2019; 48(1):537-562. doi:10.1146/annurev-biophys-070317-033330.

118. Ganzinger, K.A., Schwille, P.. More from less bottom-up reconstitution of cell biology. Journal of Cell Science 2019; 132(4):jcs227488. doi:10.1242/jcs.227488. 


\section{Figure legends}

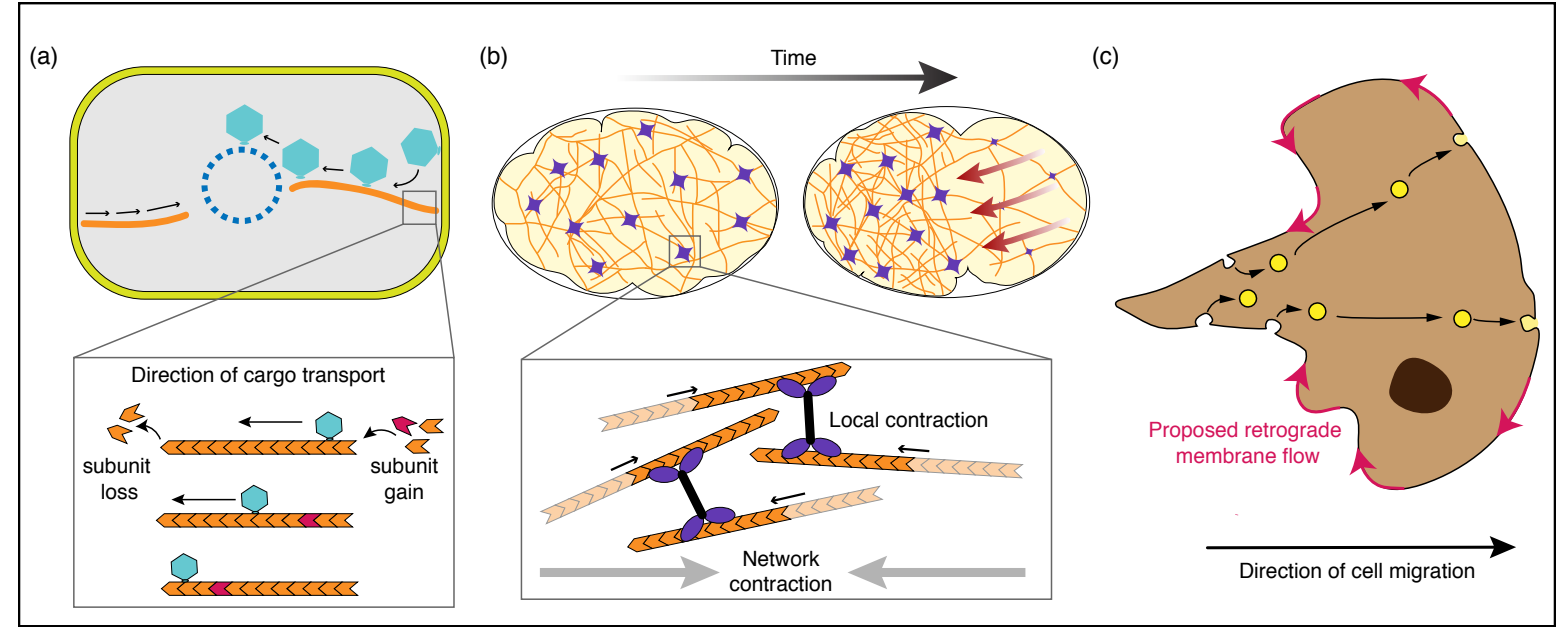

Figure 1: Types of flows (a) Treadmilling driven transport. During Pseudomonas phage infection, tubulin-like filaments (PhuZ, orange) play important roles in centering a nucleuslike structure containing phage DNA, trans port of viral capsids, and their distribution around the nuclear surface. PhuZ polymerization at the cells poles drives treadmilling and flux of subunits, which carry the attached viral capsids (blue) to the cell center. Treadmilling of PhuZ filaments also drives rotation of the phage nucleus (dark blue) to distribute arriving capsids around its surface. (b) Long range cortical flow. In the $C$. elegans zygote, cortical actomyosin flow is induced by anisotropy of network contractility. This anisotropy is caused by the sperm-donated centriole, which stimulates the local down-regulation of non-muscle myosin II activity (purple foci) at the posterior pole, resulting in anterior directed flow (red arrows) of cortical actin (orange). (c) In migrating cells, a polarized cycle of endo and exocytosis of membrane components, with exocytosis at the leading edge coupled to endocytosis at the cell rear, leads to retrograde flow of material in the bilayer (red arrows). It has been hypothesized that this membrane flow could act as a 'fluid drive' to propel the cell forward. 


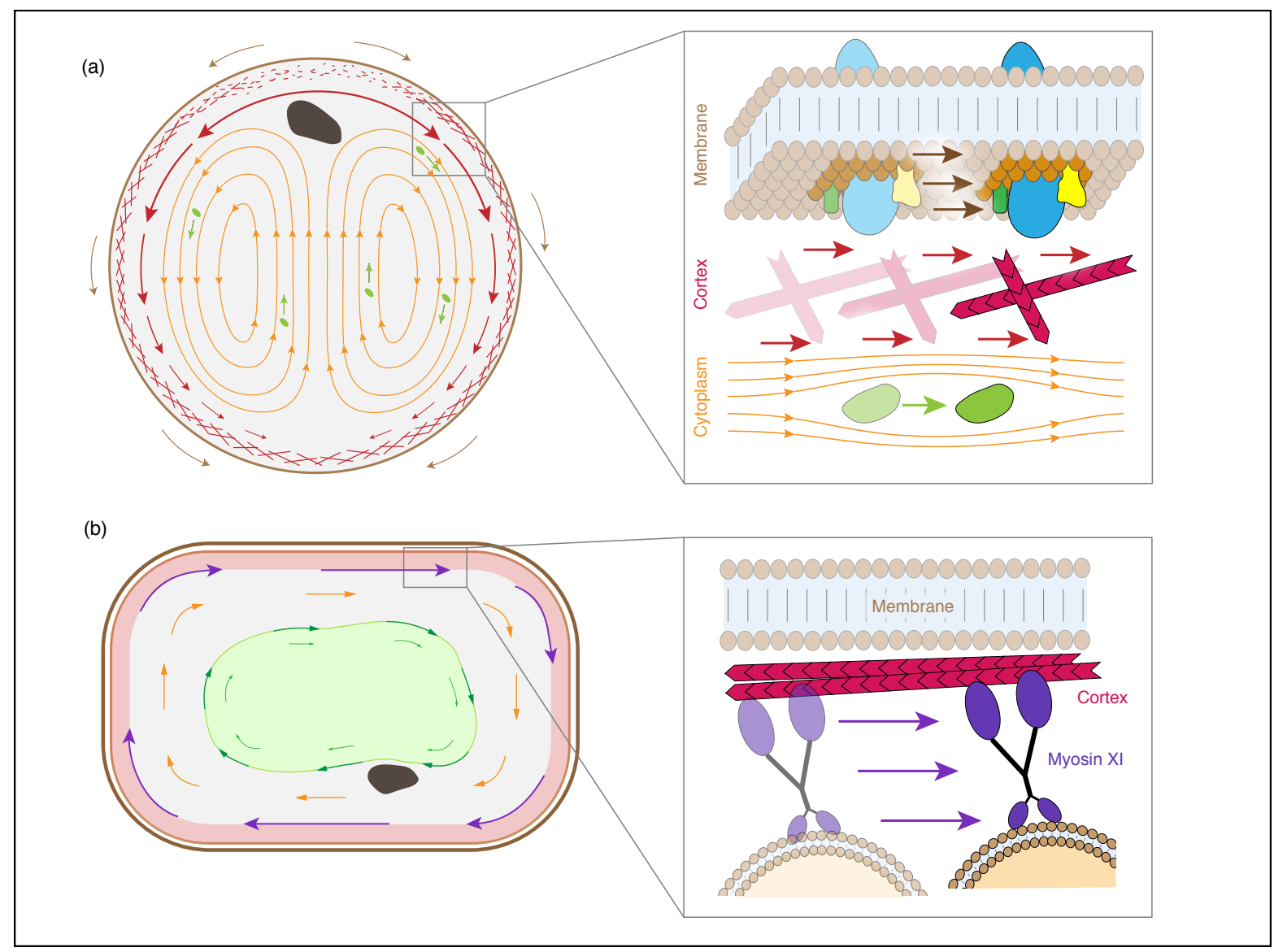

Figure 2: Viscous coupling of fluid layers. (a) Model for reverse fountain flow streaming driven by cortical actomyosin (red). Here flow of the actomyosin cortex (red arrows) generates shear stress, which is transmitted to the overlaying membrane (brown arrows) and adjacent cytoplasm (orange arrows). Motion of the cortex can therefore be coupled to flow of both cytoplasmic components (green arrows) and transmembrane proteins with their surrounding lipids (dark brown arrows, inset). (b) Model of circulatory flow (cyclosis) in a plant cell. Myosin XI (purple, inset) transports large organelles, such as the ER, along oriented cortical actin filaments (purple arrows), inducing flow of the cytoplasm (orange arrows) in which they are moving. The cytoplasm is hydrodynamically coupled to the vacuole interior (green) via the vacuolar membrane. Thus, shear stress originating from Myosin XI motion at the cell cortex propagates throughout the various compartments of the cell, driving the observed pattern of fluid flow. 


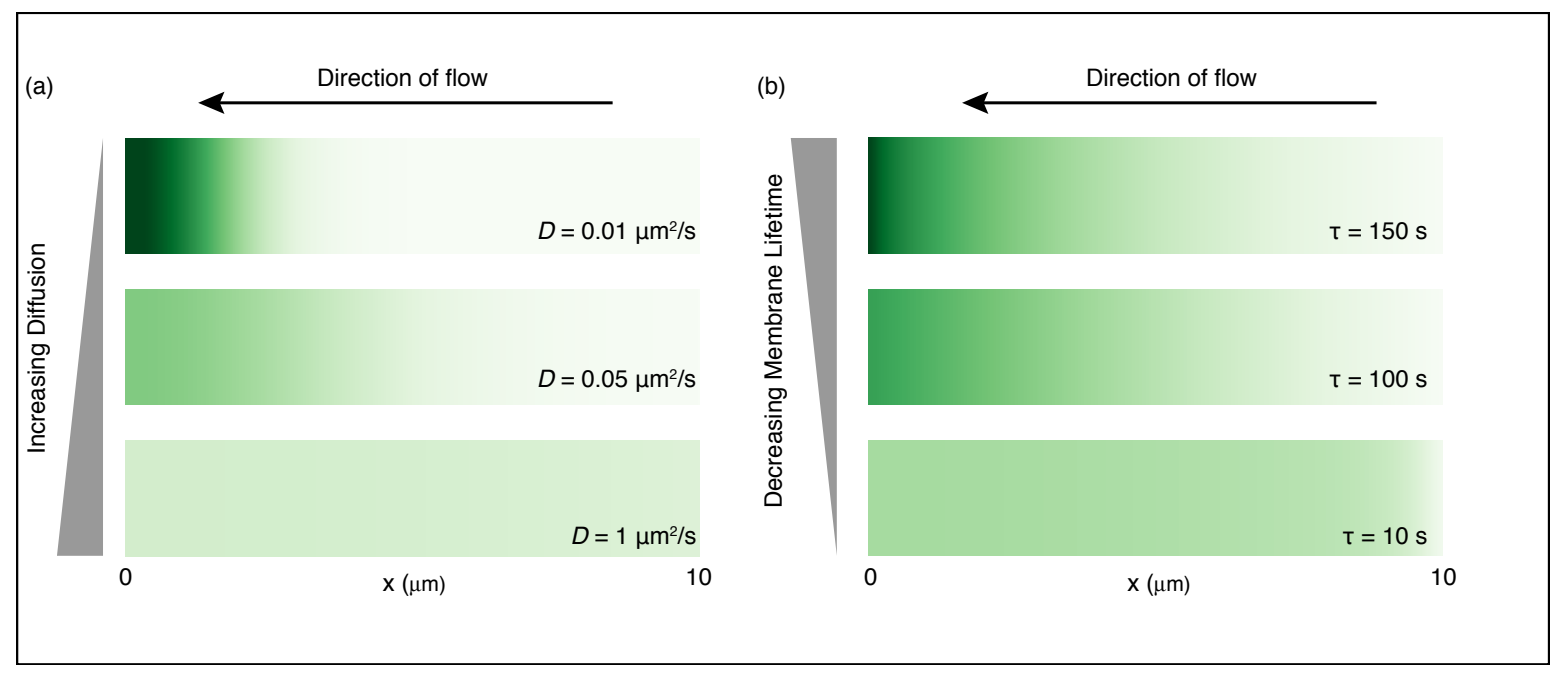

Figure 3: Flow-induced asymmetry depends on the ratio of diffusive and advective transport. For membrane-associated species subject to advection, asymmetric accumulation by flow will depend on multiple factors: flow velocity, diffusion rates on the membrane and in the cytoplasm, and the rate of exchange between the membrane and cytoplasmic compartments. Here we consider two simplified cases in which flow velocity is held constant and we vary either diffusion or membrane dissociation rates. In (a), we consider a molecule that is stably associated with the membrane (i.e. does not exchange). For a given flow velocity, asymmetry is inversely related to the diffusion coefficient gradients steepen as the diffusion is reduced (i.e. increasing $P e$ ). In (b), we consider the case of varying membrane detachment rates, holding diffusion in the two compartments fixed, in this case $\left(D_{\text {mem }}=0\right.$, $\left.D_{\text {cyto }}=1 \mu \mathrm{m}^{2} / \mathrm{s}\right)$. Here, asymmetry declines with decreasing lifetime of the bound state, as the time spent being advected is decreased relative to the time spent diffusing (i.e. decreasing $\mathrm{Pe}$ ). Plots show distributions from a 1-D simulation implementing a graded velocity function across the system where flow velocity $u=-0.005 * x(\mu \mathrm{m} / \mathrm{s})$. Concentration shown in green. 

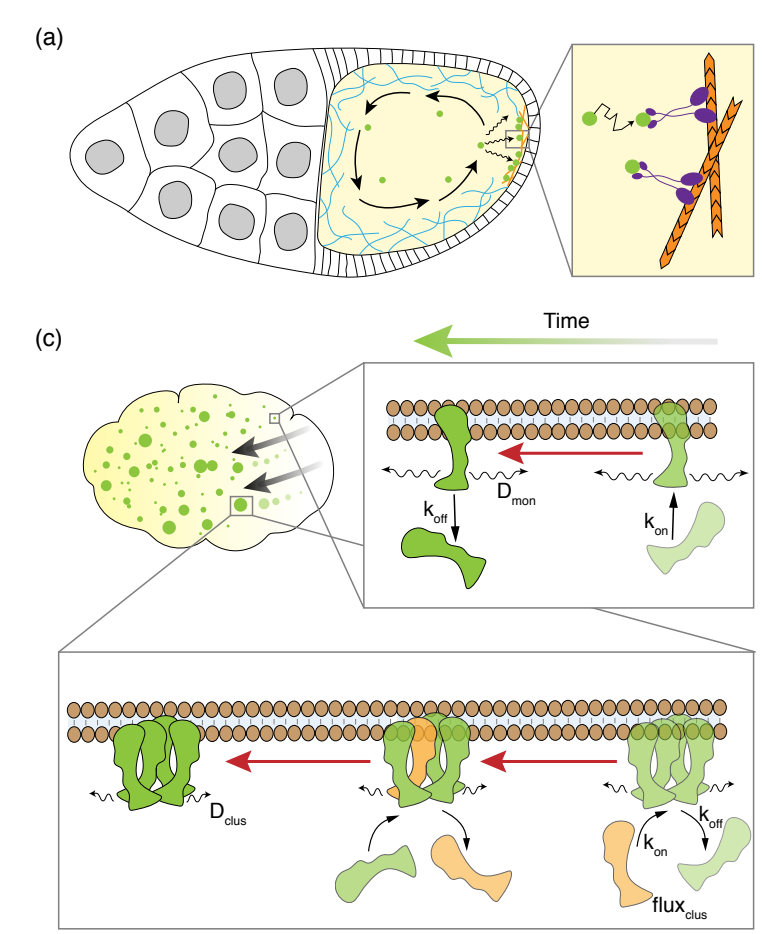

(b)

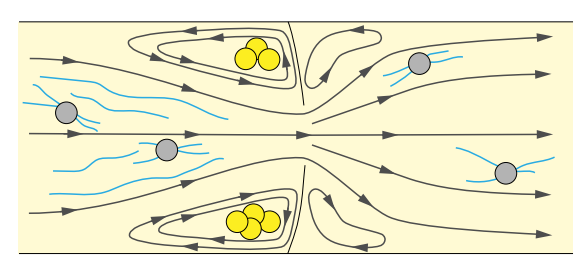

(d) (i)
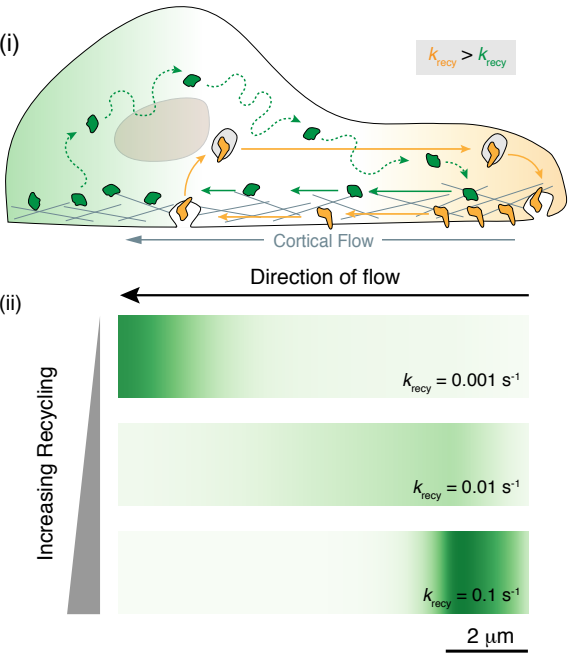

Figure 4: Examples of flow-induced polarity. (a) Kinesin-dependent microtubule streaming (MTs in blue) in the Drosophila stage 10B oocyte drives the mixing of oskar mRNA and RNA-binding proteins (green) allowing it to sample the cell cytoplasm. Inset illustrates myosin V-dependent (purple) entrapment of RNPs at the posterior cortex. (b) In the multi-nucleated fungus Neurospora crassa, hyphal filaments are compartmentalized by septa, perforated by a central pore. Bulk cytoplasm flows unidirectionally (black arrows) through these pores, generating flow vortices known as eddies in the corners of the hyphal compartments. Nuclei and other organelles become trapped in these eddies (yellow), forming aggregates in which the behavior of organelles changes. In particular, confined nuclei engage distinct develop mental programs compared to flowing nuclei (grey) that are associated with the microtubule network (blue), leading to asymmetry within the hyphal compartments. (c) In the C. elegans embryo, clustering of PAR-3 is critical for advective transport by anteriordirected cortical flow. PAR-3 monomers (top inset) are highly diffusive and exchange between the membrane and cytoplasm rapidly, making advection inefficient (low $P e$ ). By contrast, PAR-3 dependent clusters (bottom inset) remain membrane-associated much longer 
and exhibit reduced diffusion, facilitating long range transport. (d) (i) In migrating cells, retrograde actin flow transports molecules towards the rear end of the cell. The accumulation of molecules at the rear by flow is opposed by turnover at the membrane which allows molecules to be recycled back to the cell front. A fast rate of turnover and recycling coupled to local deposition at the leading edge allows concentration at the cell front (yellow species), while slow turnover (green) drives accumulation at the rear. (ii) Altering turnover in a simple mathematical model of advective transport dramatically alters the concentration profile of molecules across the cell. Here a slowly diffusing molecule on the membrane $(D=0.01$ $\mu \mathrm{m}^{2} / s$ ) is subject to flow as in Figure 3 and recycled to the cell front at variable rates $k_{\text {recy. }}$. 